\title{
Hector: a high-multiplex survey instrument for spatially-resolved galaxy spectroscopy
}

\author{
Jon Lawrence ${ }^{a, *}$, Joss Bland-Hawthorn ${ }^{b}$, Julia Bryant ${ }^{b}$, Jurek Brzeski ${ }^{a}$, Matthew Colless ${ }^{a}$, \\ Scott Croom $^{b}$, Luke Gers $^{a}$, James Gilbert ${ }^{a}$, Peter Gillingham ${ }^{a}$, Michael Goodwin ${ }^{a}$, Jeroen Heijmans ${ }^{a}$, \\ Anthony Horton ${ }^{a}$, Mike Ireland ${ }^{a, c}$, Stan Miziarski ${ }^{a}$, Will Saunders ${ }^{a}$, Greg Smith ${ }^{a}$ \\ ${ }^{a}$ Australian Astronomical Observatory, PO Box 296, Epping, NSW 2121, Australia \\ ${ }^{b}$ School of Physics, The University of Sydney, NSW 2006, Australia \\ ${ }^{c}$ Department of Physics and Astronomy, Macquarie University, NSW 2109, Australia
}

\begin{abstract}
First light from the SAMI (Sydney-AAO Multi-object IFS) instrument at the Anglo-Australian Telescope (AAT) has recently proven the viability of fibre hexabundles for multi-IFU spectroscopy. SAMI, which comprises 13 hexabundle IFUs deployable over a 1 degree field-of-view, has recently begun science observations, and will target a survey of several thousand galaxies. The scientific outputs from such galaxy surveys are strongly linked to survey size, leading the push towards instruments with higher multiplex capability. We have begun work on a new instrument concept, called Hector, which will target a spatially-resolved spectroscopic survey of up to one hundred thousand galaxies. The key science questions for this instrument concept include how do galaxies get their gas, how is star formation and nuclear activity affected by environment, what is the role of feedback, and what processes can be linked to galaxy groups and clusters. One design option for Hector uses the existing 2 degree field-of view top end at the AAT, with 50 individual robotically deployable 61-core hexabundle IFUs, and 3 fixed format spectrographs covering the visible wavelength range with a spectral resolution of approximately 4000. A more ambitious option incorporates a modified top end at the AAT with a new 3 degree field-of-view wide-field-corrector and 100 hexabundle IFUs feeding 6 spectrographs.
\end{abstract}

Keywords: integral field spectroscopy, astronomical spectroscopy, IFU, fibre positioning systems, wide field correctors

\section{INTRODUCTION}

Until recently, major surveys of galaxies have been restricted to single-aperture spectroscopy. Optical spectroscopy allows us to measure a wide range of parameters including current star formation rates, gas phase metallicities, stellar ages, stellar metallicities, black hole accretion rates, ionization structure and extinction due to dust. But the singleaperture measurements can be biased depending on where the aperture falls on the image of the galaxy [1].

Over the last decade, projects such as SAURON [2] have demonstrated the power of integral field spectroscopy to capture a range of key observables that are simply not available to single-aperture spectroscopic surveys. As well as studying the properties listed above in a spatially-resolved context, obtaining gas and stellar kinematics over an entire galaxy enables us to separate dynamical components, measure dynamical masses, examine the impact of winds and outflows, and discover merging systems via dynamical effects. Integral field spectroscopy has been limited to singleobject instruments, meaning that it is time-consuming to build large samples. The largest current data set using the SAURON system on the $4.2 \mathrm{~m}$ William Herschel Telescope contains 260 objects (ATLAS-3D; [3]). The CALIFA project [4] aims to target $\sim 600$ objects with the PMAS IFS on the $3.5 \mathrm{~m}$ Calar Alto Telescope using $~ 200$ nights of telescope time.

The recently developed SAMI instrument [5] at the Anglo-Australian Telescope (AAT) comprises 13 hexabundle IFUs that are positioned using a manual plug-plate at the 1 degree FOV triplet top end feeding the AAOmega spectrograph [6]. SAMI should be capable of performing surveys up to several thousand objects, e.g., 100 nights $\mathrm{x} 4$ fields/night $\mathrm{x} 13$ objects/field x 50\% efficiency $=2600$ objects. Here we propose the Hector instrument concept that aims to eclipse existing surveys through a 5-10-fold increase in sample size relative to SAMI. Two baseline options for Hector are considered: one consisting of 52 IFUs that are positionable over a 2 degree FOV, the other consisting of 117 IFUs that are positionable over a 3 degree FOV.

*j1@aao.gov.au

Ground-based and Airborne Instrumentation for Astronomy IV, edited by lan S. McLean, Suzanne K. Ramsay, Hideki Takami, Proc. of SPIE Vol. 8446, 844653 - ( ) 2012 SPIE · CCC code: 0277-786X/12/\$18 · doi: 10.1117/12.925260 

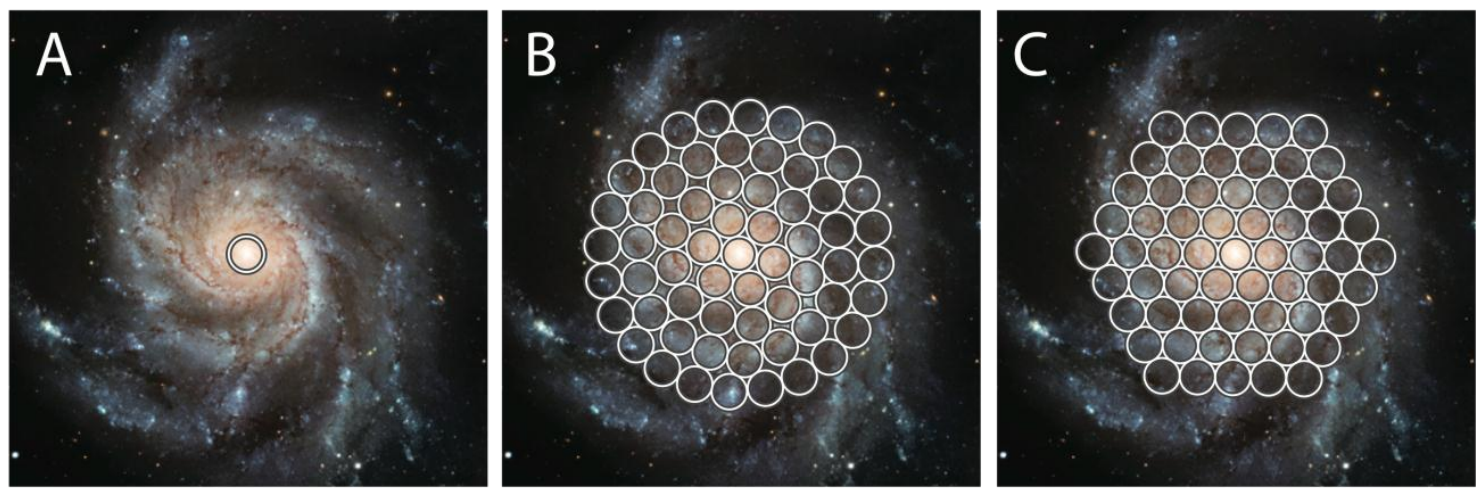

Figure 1. (a) single fibre apertures are the basis for galaxy redshift surveys to date; (b) the 61-fibre configuration of the hexabundle used in the SAMI instrument; (c) an improved configuration that is better optimized for "dithering" (i.e. small shifts with respect to sky to remove gaps between fibres) and to greatly simplify data analysis.

\section{SCIENCE}

The Hector survey of $\sim 10^{4}-10^{5}$ galaxies will deliver a transformational new view of how galaxy properties vary across a contiguous volume of the universe. The survey will initially target Stripe 82 from the Sloan Digital Sky Survey which is a region of the sky with existing multiband optical photometry. The huge database will provide information on galaxy properties, in particular, stellar and gas kinematics, the presence of nuclear activity (starburst vs. black hole), the star formation distribution, the spread of chemical abundance in both stars and gas, and so forth. The "Environments of Galaxies" survey is made up of a number of sub-projects including:

1. How do galaxies get their gas?

2. How is star formation and nuclear activity affected by environment?

3. What is the role of feedback?

4. Galaxy groups and clusters

The survey bears on one of the most pressing questions and one of the great mysteries of modern astrophysics: "How does gas move in and out of galaxies over cosmic time?" The answer to this question is fundamental to the origin and evolution of galaxies. This gas is the main ingredient of star formation which in turn produces the chemistry we observe in stars and gas. The new survey will address one of cosmology's most important questions "How did our Milky Way galaxy form?" and help to elucidate the role of the unseen dark matter.

\subsection{How do galaxies get their gas?}

Before the advent of supercomputers, galaxy formation theory assumed that gas collapsed under gravity and consequently became shock heated. If there was enough local gravity to hold onto the hot gas, e.g. from dark matter, it would eventually cool and form stars. But in recent years, both observations and models show that this cannot be the dominant mode of gas accretion onto galaxies. A related theory is that the gas falls in with the accreting dark matter but simulations consistently fail to reproduce the majority of luminous galaxies which are characterized by thin stellar disks.

Kormendy et al [7] have shown that two thirds of their nearby galaxy sample are pure disks with only small central bulges and found no correspondence with the big-bulge thick disks arising naturally in the simulations. These are simply too violent to form the thin disks we observe today predicting very high merger rates of infalling dark matter clumps and too many clumps at all epochs. The gathering consensus is that gas must separate from dark matter on large scales and enter a disk galaxy quietly. Most of the gas must enter disk galaxies in a "cold" or a "warm" flow over billions of years of cosmic time. Newly contrived models that use quiescent gas accretion do seem to produce better looking galaxies.

A simple test of gas accretion models is to see if the spin properties of galaxies are correlated or not at all. The extent to which properties are correlated will determine whether gas is mostly confined by dark matter over cosmic time, or 
whether the gas became separated at an earlier epoch and has gently flowed back since then [8]. Before the development of multiplexing spatially resolved spectroscopic instruments, a large-scale survey of this kind was inconceivable.

\subsection{How is star formation and nuclear activity affected by environment?}

Some of the most energetic galaxies in the universe are powered by a combination of starburst and black-hole activity at their central nuclei. It is now believed that these nuclear phenomena experience a complex synergistic relationship through cosmic time [9]. Black hole growth benefits from its association with extreme star-formation activity, that is mediated by outbursts from the accretion disk around the black hole. The integral field outputs from the Hector instrument will allow us to disentangle the influence of nuclear AGN (including the mysterious LINER activity; [10]) and star formation activity within each object. It may well be that some environments favour AGN activity over star formation, and vice versa. Hector will provide important information about their synergistic relationship with the density of the local environment.

\subsection{What is the role of feedback?}

Feedback from star formation and accretion onto supermassive black holes provides an internal mechanism for transformation. This feedback provides a solution to the mismatch of the theoretical dark matter halo mass function and the observed stellar mass function by heating and/or expelling gas in both low mass (via star formation) and high mass (via black hole accretion) haloes. Extreme outbursts of star formation or black hole accretion may be triggered by mergers or interactions making a link between internal and environmental effects. Once the burst is over, another mechanism is needed to suppress continued star formation. The best suggestion for this is mechanical feedback from jets emitted by supermassive black holes (e.g. [11]), but this only appears to be efficient in massive galaxies.

Recently, Sharp \& Bland-Hawthorn [12] have shown how starburst-driven and AGN-driven winds can be distinguished due to the different ionization state of the outflowing gas. Remarkably, a powerful new galactic wind source was discovered in the first 13 objects observed using the Sharp \& Bland-Hawthorn [12] diagnostic. The existence of the wind could never have been discerned from a single fibre observation of this galaxy emphasizing the extraordinary power of multiplexed spatially resolved spectroscopy. Hector may reveal a higher fraction of winds across the entire CDM hierarchy thereby allowing us to uncover which environments favour winds and which galaxy properties are affected by winds and other feedback processes.

\subsection{Galaxy groups and clusters}

The environment of a galaxy has a profound impact on its formation history and this is most clearly evident in groups and clusters. This has been demonstrated time and again through a wide variety of observations. Red sequence galaxies are preferentially found in denser environments, and star formation is also clearly suppressed at high density. This immediately suggests environmental factors play an important role. Much recent observational and theoretical work has focused on how blue galaxies can have their star formation quenched, moving them onto the red sequence. When a galaxy falls into a cluster, the ram pressure from the dense intergalactic medium [13] may expel the gas from the disc, removing the fuel required for further star formation. There are several observed examples of this in rich clusters. In moderately dense regions, such as galaxy groups, ram pressure may leave the disc intact, but can still remove gas from the halo of the galaxy. The halo provides a reservoir of gas which can replenish the disc. In addition to star formation activity, the ATLAS-3D survey has revealed that galaxy kinematics also depend on environment, with dispersionsupported early-type galaxies (slow rotators) being more common in the highest density regions.

Despite the wealth of information at hand, the physical processes at play are still hard to discern. The fact that the environmental trends extend to relatively low density implies that it is not only cluster specific processes such as rampressure stripping which influence star formation. Much of the transition could occur in group environments. While processes which control the flow of gas can modulate star formation, dynamical processes (such as galaxy merging) are required to dramatically change galaxy kinematics. Almost all analyses of environment have been conducted with singleaperture fibre surveys. But these surveys miss any spatially resolved information and the spectral information they do contain is biased by the location of the fibre on the galaxy in question [14]. A large Hector survey is needed to disentangle issues of ram pressure stripping, tidal interaction and other competing processes. 


\section{INSTRUMENT CONCEPT}

\subsection{Hector corrector}

The available field of view for the Hector instrument crucially determines the science outputs and the instrument configuration in terms of the number of fibres and IFUs allowed, and hence the number and configuration of the spectrographs.

The first option considered for Hector is to use the existing $2 \mathrm{dF}$ corrector at the AAT. This corrector was constructed for use with the $2 \mathrm{dF}$ fibre positioning system, is currently used with the AAOmega spectrograph, and will be used for the HERMES instrument now under construction. The $2 \mathrm{dF}$ corrector consists of 6 lenses with 8 air/glass interfaces. It provides $\sim 1 "$ images over a $2^{\circ}$ diameter field of view [15]. $2 \mathrm{dF}$ has an atmospheric dispersion compensator (ADC) built into the front two elements of the corrector. These two elements are slightly prismatic and are automatically counterrotated during observations to provide an equal but opposite dispersion, to counteract the atmosphere as the telescope tracks across the sky.
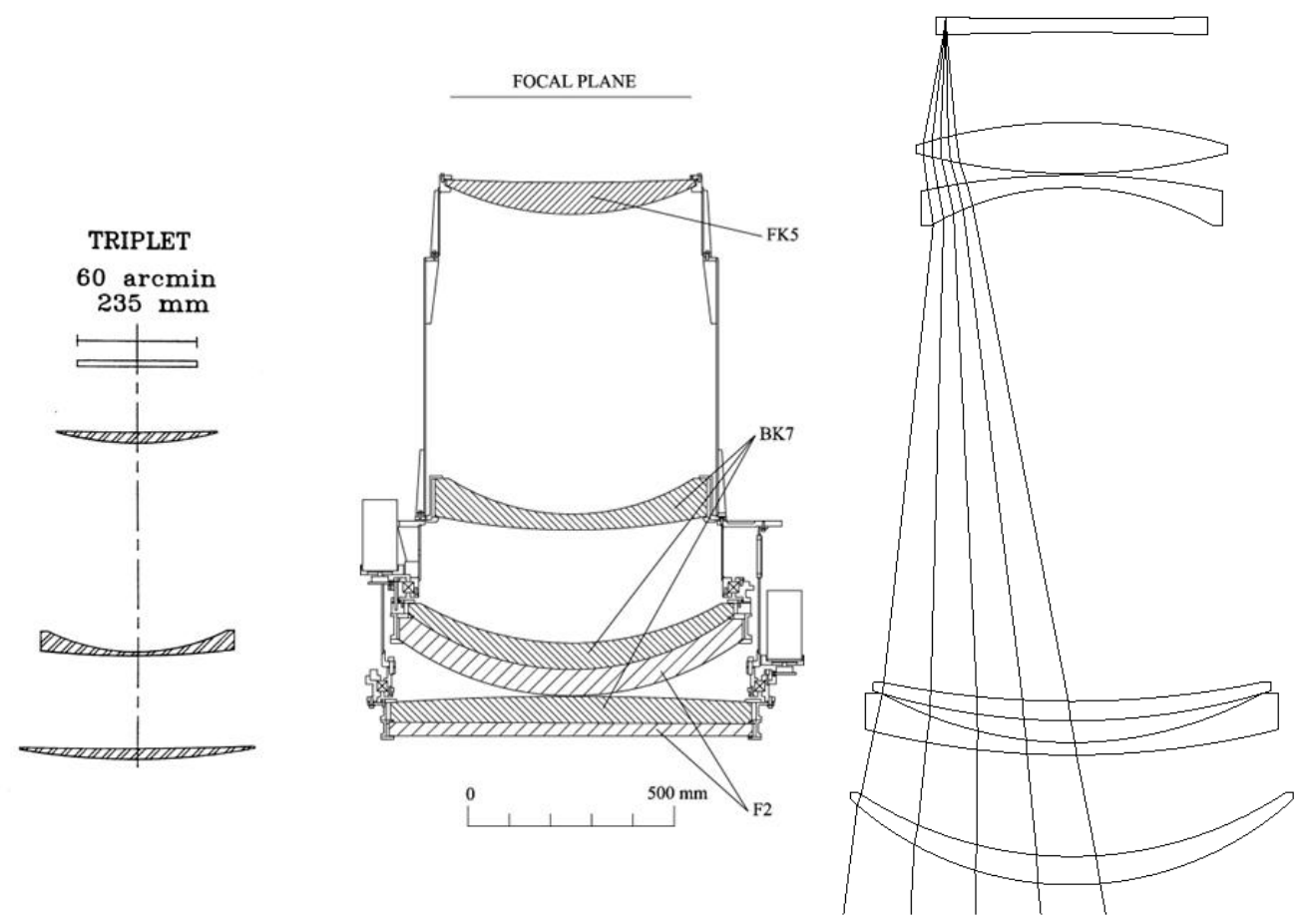

Figure 2. The optical layout of the existing 1 degree FOV triplet (left), the existing $2 \mathrm{dF}$ corrector (middle), and the design for the $3 \mathrm{dF}$ corrector (right). Figures are approximately to relative scale.

Motivated by the Hector instrument concept, we have investigated the potential for significantly widening the AAT field of view with a new corrector. This preliminary study [16] has demonstrated that very satisfactory performance is possible for feeding IFUs with a reasonably simple design, over a $3^{\circ}$ diameter field. The high level requirements for this corrector were to achieve $80 \%$ enclosed energy within 1 " diameter over a wavelength range of $400-900 \mathrm{~nm}$ with a telecentric focal plane. In comparison with the existing $2 \mathrm{dF}$ corrector, the $3 \mathrm{dF}$ corrector gives less smear of images due to chromatic aberration combined with atmospheric dispersion for zenith angles less than about $30^{\circ}$. Beyond $30^{\circ}$, the $2 \mathrm{dF}$ corrector progressively gains through using an ADC. Note, however, that this atmospheric chromatic dispersion can be post-corrected within each IFU for Hector. Figure 2 shows the optical layout for the triplet corrector (currently used by the SAMI prototype), the $2 \mathrm{dF}$ corrector (used by AAOmega), and the proposed new $3 \mathrm{dF}$ Hector corrector.

\subsection{Telescope top-end configuration}

There are two different locations for the Hector IFU positioning unit depending on which of the $2 \mathrm{dF}$ or $3 \mathrm{dF}$ concepts is chosen. If the $2 \mathrm{dF}$ corrector is used then the Hector IFU positioner must be compatible with the existing $2 \mathrm{dF}$ top-end at 
the AAT. This top-end (shown in Figure 3) consists of the corrector and ADC mounted within a central structure onto a top-end ring that interfaces to the telescope Serrurier ring. Above the corrector, a tumbler is used to switch focal plates between a (lower) observing field and an (upper) configuring field. The positioner and gripper robot is located at the top of the centre structure. The Hector $2 \mathrm{dF}$ instrument top-end would have to interface to this structure. There are several options for this interface depending on the positioning system used (see next section).

For the $3 \mathrm{dF}$ Hector version, the original telescope prime focus top-end could be modified (see Figure 3). In this case the top end outer ring with its provisions for semi-automatic attachment to the telescope Serrurier ring would be re-used but the central structure and the eight vanes connecting it to the outer ring would be replaced. The central structure would house the new corrector and the new Hector IFU positioning unit. In comparison with fitting a robotic multi-IFU system on the $2 \mathrm{dF}$ top end, fitting it together with the $3 \mathrm{dF}$ corrector as discussed here would cause much less disruption to AAT operations during Hector integration. It would also avoid compromising $2 \mathrm{dF}$ operation through over-crowding and complication of that top end.
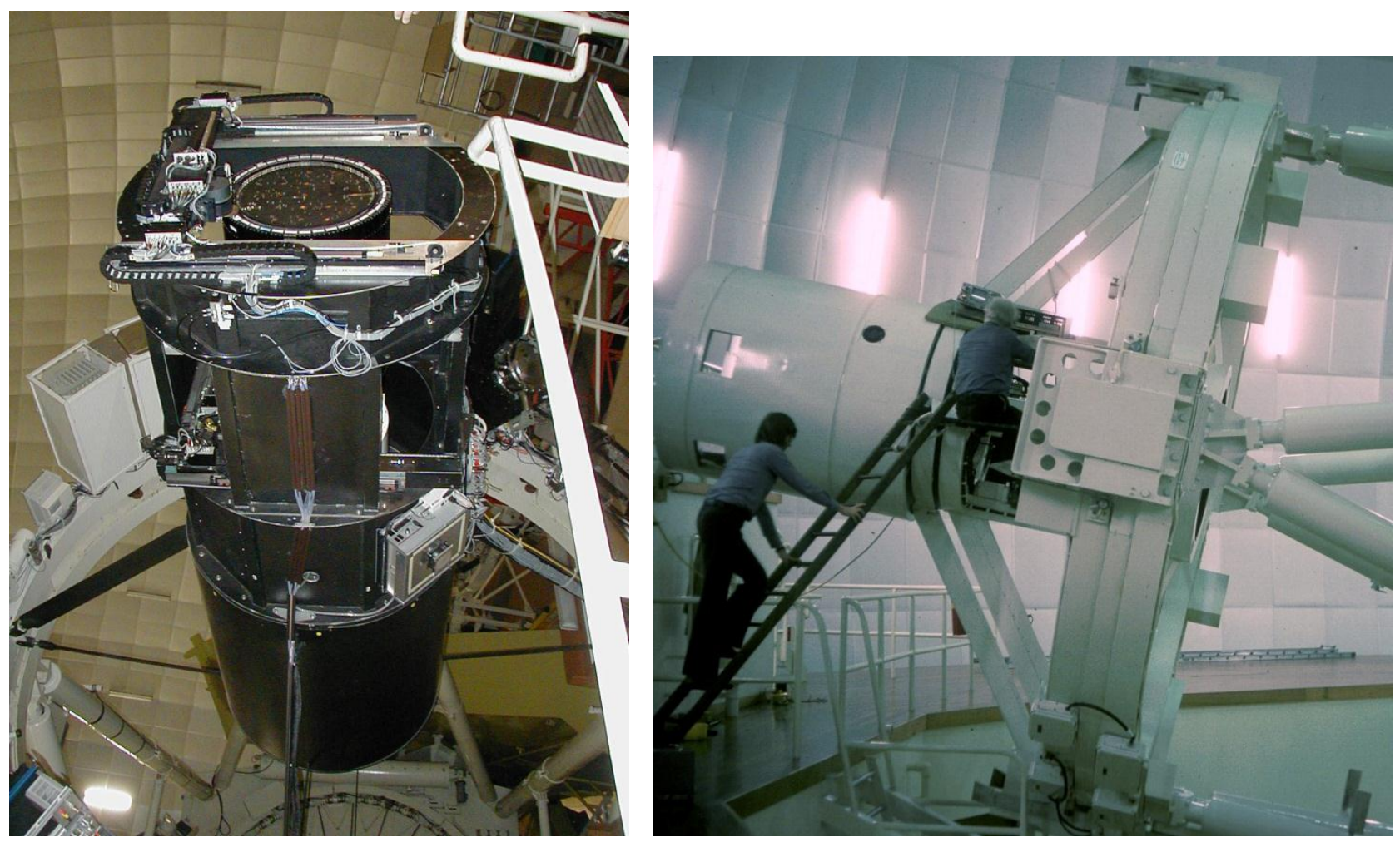

Figure 3. Left: $2 \mathrm{dF}$ field plate and robot mounted with the corrector and tumbler structure in the AAT 2dF top-end ring.

Right: View of original prime focus top end (during commissioning of the telescope auto guider), shown attached to the telescope top-end ring.

\subsection{Positioning system}

The following are the high level requirements for the Hector IFU positioning system:

- The positioning system shall locate IFUs at the focal plane at the AAT top end using either the existing $2 \mathrm{dF}$ top end or a new $3 \mathrm{dF}$ top end.

- The total number of IFUs will be $\sim 60$ for the $2 \mathrm{dF}$ version or $\sim 100$ for the $3 \mathrm{dF}$ version.

- An extra $\sim 15$ or 40 (for $2 \mathrm{dF}$ or $3 \mathrm{dF}$ respectively) positions for sky fibres are needed for sky subtraction. It is likely that these need to be independently positioned.

- Nominally, each IFU will consist of a hexabundle with 61 fibres in a close-packed lightly fused head with 100 micron cores. It is possible other hexabundle types (eg, 37, 91 core) will also be included. Each hexabundle will be housed in a stainless steel tube that is $\sim 4-6 \mathrm{~mm}$ diameter and 50-80 mm length. 
- An alternative to hexabundles would be to construct each IFU from fibres bonded to the rear of a pair of microlens arrays with a relay lens in front. This assembly would be housed within a tube that is $6-10 \mathrm{~mm}$ in diameter and $20-40 \mathrm{~mm}$ length.

- The position tolerance for each IFU in $\mathrm{x}, \mathrm{y}$ is $\pm 25 \mu \mathrm{m}$.

- The position tolerance for each IFU in $\mathrm{z}$ is $\pm 100 \mu \mathrm{m}$.

- The tolerance in tip/tilt of each IFU head wrt to the focal plane is \pm 0.5 degrees.

- Each IFU shall be aligned with a rotation axis that is measurable to +/-5 degrees.

With the above set of requirements, we have investigated a series of different options for positioning systems. Options have included: manual plug-plates, Echidna (spine) style actuators, 2dF pick-and place style, X-theta robots, Starbugs, pick-off-mirrors, fisherman style, chess robot, fixed patrol field, and magnetic floating buttons. See [17] for more details on these positioning system concepts. The outcomes of our studies have suggested that for the $3 \mathrm{dF}$ version of Hector, Starbugs is the preferred positioning technology, and for the $2 \mathrm{dF}$ version of Hector, a sequential robot (either a pick-andplace robot with mechanical gripper or a suck-and-slide robot with a magnetic floating button) is the preferred positioning technology.
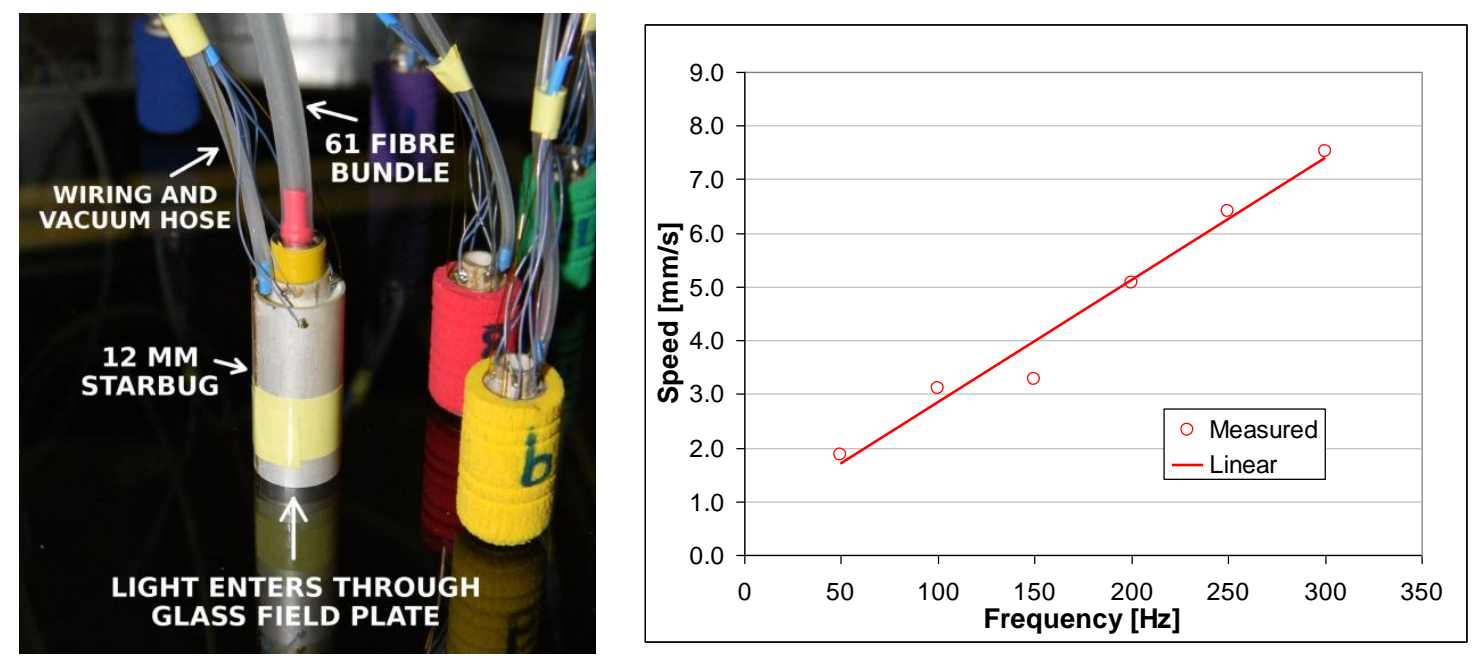

Figure 4. Left: Prototype $12 \mathrm{~mm}$ Starbug (centre of picture) carrying a 61 fibre bundle on a horizontal glass field plate. The smaller Starbugs in the picture are designed for small (7 or 19 element) IFUs. Right: Measured movement speed for a 12 $\mathrm{mm}$ Starbug on a horizontal glass field plate; frequency corresponds to the rate of stepping ( $300 \mathrm{~Hz}$ is current system maximum).

Starbugs are robots that can simultaneously position many optical payloads on a telescope's focal plane and rotate them to any angle [18]. They are currently being developed for the MANIFEST (many instrument fibre system) instrument for the Giant Magellan Telescope, which will nominally require positioning of many small (e.g. 19 fibre) bundles [19]. Figure 4 shows a prototype large Starbug for positioning heavier payloads such as hexabundles. Its $12 \mathrm{~mm}$ diameter is necessary because Starbugs adhere to a field plate using an internal vacuum. Increasing the area under vacuum means that the adhesion force is also increased and therefore larger loads can be moved. The Starbugs position on a transparent field plate, with light passing through the plate and into the central aperture of the Starbug where the IFUs are mounted.

Initial results show good performance for a large Starbug moving a bundle of 61 fibres across a horizontal field plate (Figure 4). In this case, the Starbug was able to position to an accuracy of a few microns under closed-loop control. The Starbug's performance deteriorates as the field plate is moved towards vertical (which may have a minor impact on field configuration times), although they remain attached to the plate for all orientations. Further work must be done to fully optimise a Starbug system for use with these large payloads, although successful positioning on a horizontal field plate proves that Starbugs and hexabundles are compatible. If the IFU comprises dual microlens arrays (rather than hexabundles) then Starbugs are also compatible as that is the preferred IFU configuration for the MANIFEST instrument for which the Starbugs system has been developed. 
For the $2 \mathrm{dF}$ version of Hector, the sequential robot is preferred over Starbugs because the $2 \mathrm{dF}$ Hector system must interface opto-mechanically with the existing $2 \mathrm{dF}$ top-end, and it is not clear there is enough space available for Starbugs. Additionally, using the same or similar positioning technology as for the $2 \mathrm{dF}$ robot could potentially simplify the system. Options considered (see Figure 5 and [17]) include using the existing $2 \mathrm{dF}$ robot with either a slightly modified mechanical gripper (for pick-and-place operation) or a new pneumatic gripper (for suck-and-slide operation), and placing the Hector field plate at one of the currently spare side positions within the $2 \mathrm{dF}$ tumbler. The disadvantage of this approach is that the IFUs must be mounted horizontally with a prism on their end faces, and a fibre bundle retractor system must be included. The fibre bundle must also be isolated and not interfere with the operation of the AAOmega or HERMES fibre bundles.
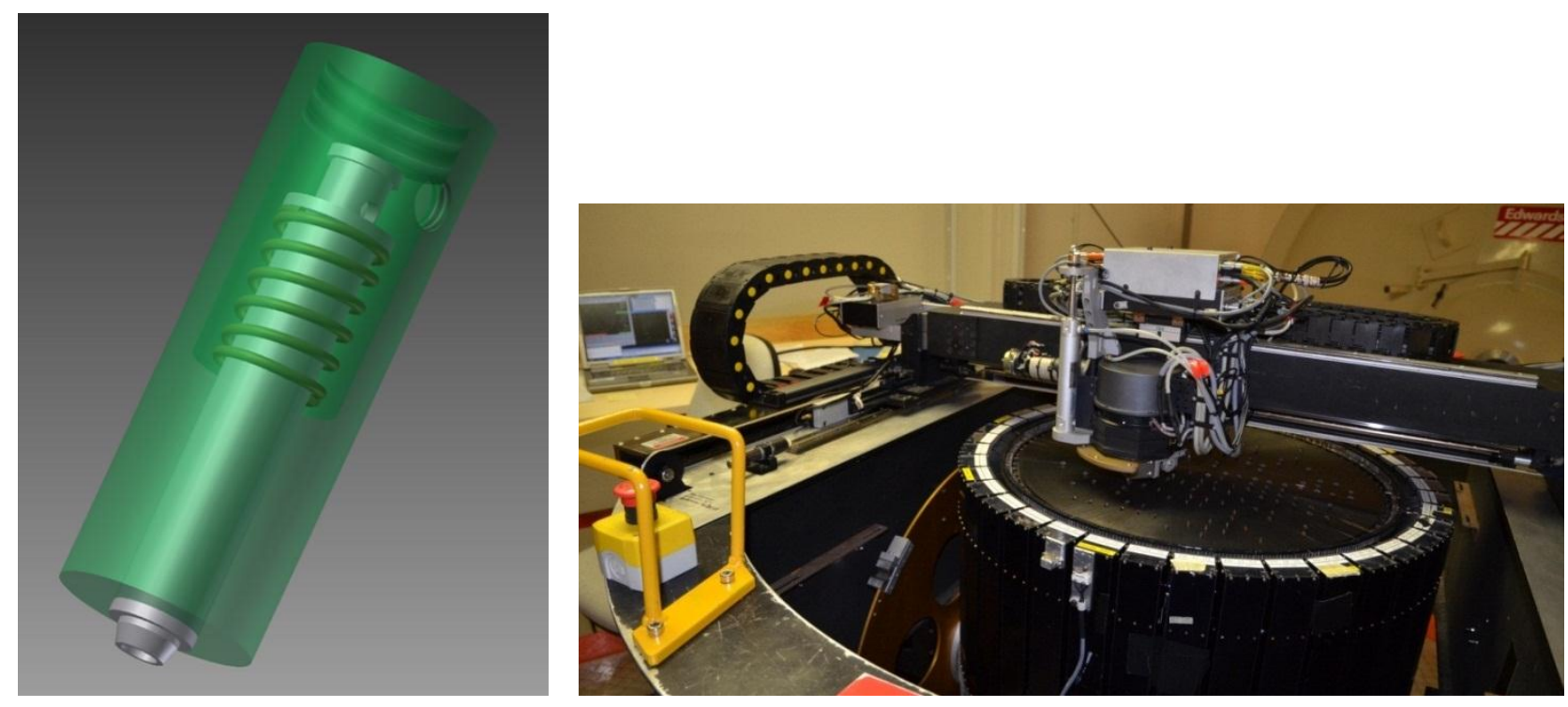

Figure 5. Left: designs for a pneumatic fibre button manipulator. In this design the button is attached to the field plate using a magnet. The robot engages and injects compressed air into the button thus forming a magnet preloaded air bearing allowing virtually friction-less repositioning of the button via the gantry. Right: $2 \mathrm{dF}$ positioning robot with mechanical gripper.

\subsection{Integral field units}

The baseline design for Hector uses hexabundle IFUs that are similar to those used in the SAMI instrument [5]. For SAMI there are 13 units, each of which comprises a 61 core hexabundle as shown in Figure 6, with individual core diameters of $105 \mu \mathrm{m}$. Each IFU thus covers a field-of-view of $\sim 15^{\prime \prime}$ with $1.6^{\prime \prime}$ sampling. This sampling is relatively close to the median seeing at Siding Spring. The size of each spatial element for Hector will likely be similar, though a full trade study will be conducted to determine the optimum size. For the majority of science cases proposed the galaxy size for a given survey is also appropriate. The instrument will likely benefit, however, from the addition of a number of larger sized IFUs (e.g., 127 cores $=22 "$ FOV; $169=26 "$ FOV). The exact details of the IFU sizes and numbers will be decided only after further development of the Hector science cases, and will be informed by the results from the initial SAMI surveys.

The key motivations for using hexabundles as IFUs are that they are relatively straightforward to manufacture at a reasonable unit cost, that they provide a simple interface (as there are no required fore-optics), and their performance is well understood (in terms of focal ratio degradation and throughput) [20,21]. The key disadvantage to the hexabundle approach is that the fill factor is not as high as would be preferred. For SAMI hexabundles the fill factor is $\sim 73 \%$. While in principal this can be increased (by reducing the fibre cladding thickness), the cross-talk increases when this is done. 

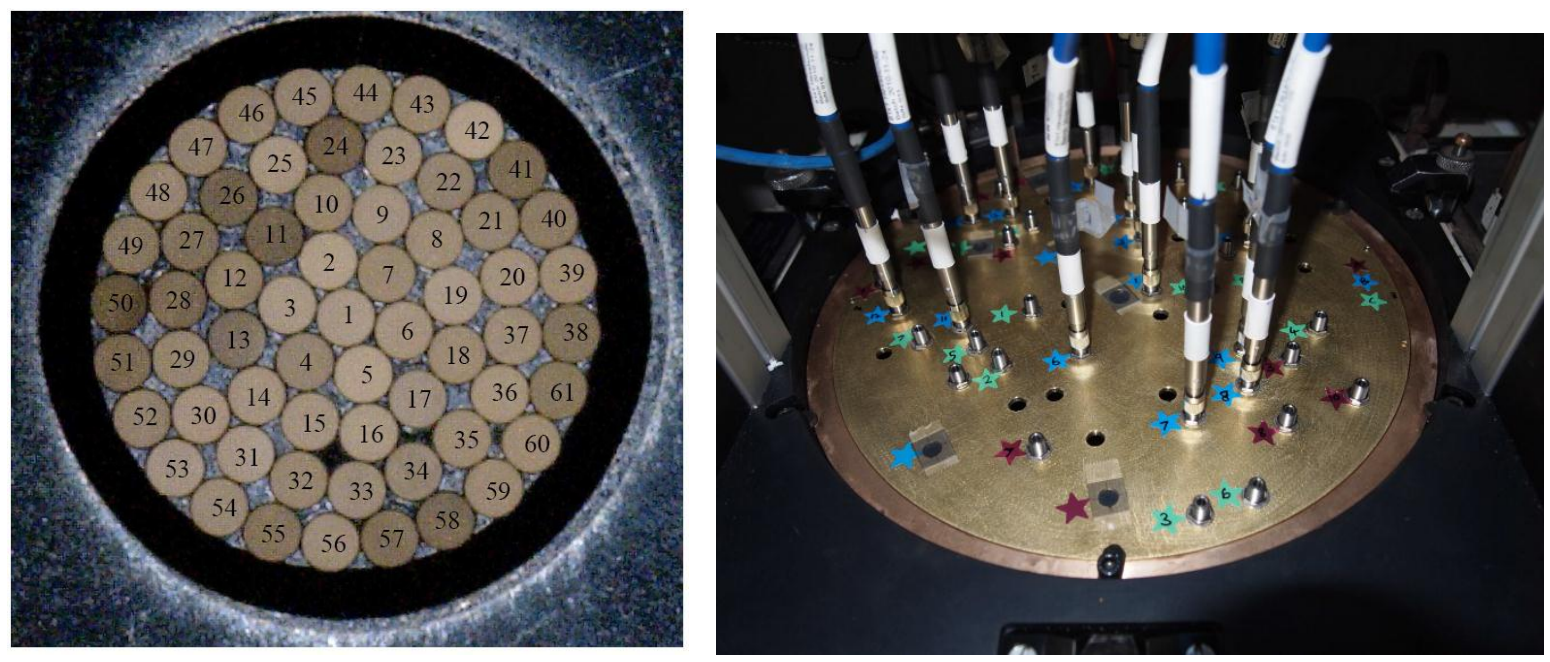

Figure 6. Left: front face of one of the 61-core fused hexabundles used in the SAMI instrument. The cores are $105 \mu \mathrm{m}$ in diameter. The fibres were back-illuminated randomly. Right: The SAMI hexabundles with steel protective tubes installed in the plug-plate at the AAT top-end.

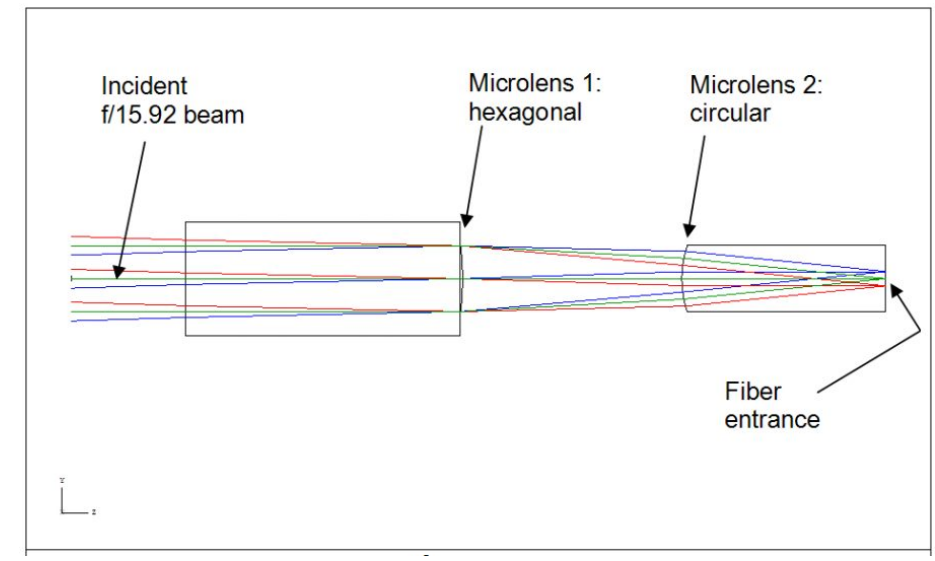

Figure 7. Dual-microlens based IFU optical design. A single microlens array element is shown for both front (hexagonal) and rear (circular) arrays. Note the telecentric injection: the center ray of all three colored pupil locations are horizontal, and the range of angles that the fiber is injected at is the same at all locations on the fiber face. This design is for an $\sim \mathrm{f} / 16$ injection. For Hector we would use a lens to convert prime focus $\mathrm{f} / 3.3$ to $\sim \mathrm{f} / 8$ before injection into the microlens.

An alternative approach to IFU construction uses a pair of microlens arrays as illustrated in Figure 7, with a free space region in between. This concept has been proposed for the KOALA [22], GHOST [23], and MANIFEST instruments [19]. A fibre array is bonded to the rear flat face of the final microlens array. In this arrangement, the two microlenses are required because the fibre is large relative to the microlens pitch $(250 \mu \mathrm{m})$. A second powered surface is thus required to preserve telecentricity in the beam from the telescope primary mirror to avoid substantial losses due to geometric focal ratio degradation. In principal no further optics are required. The microlens pitch can be made somewhat smaller $(\sim 150 \mu \mathrm{m})$, but as this corresponds to $2.2^{\prime \prime}$ would undersample the seeing limited PSF. To maintain appropriate sampling, a relay lens would be required in front of the first microlens, which would add two more air/glass interfaces.

The key advantage of the microlens-based IFU is that very high fill factors are possible (>98\%) using low scattered light hexagonal lens arrays with a hexagonal lattice. However, extra losses in throughput and/or etendue are incurred, due to the extra surfaces and absorptions, the hexagonal beam, and alignment errors. Such microlens based IFUs have already been prototyped (see Figure 8). While designs exist (also in Figure 8), further work is needed to miniaturise the microlens assembly so that it can be interfaced to a single Starbug. The IFU type selected for Hector (whether hexabundle or microlens-based) will be informed by a future trade study, considering the field of view and fill-factor requirements for key science cases. 

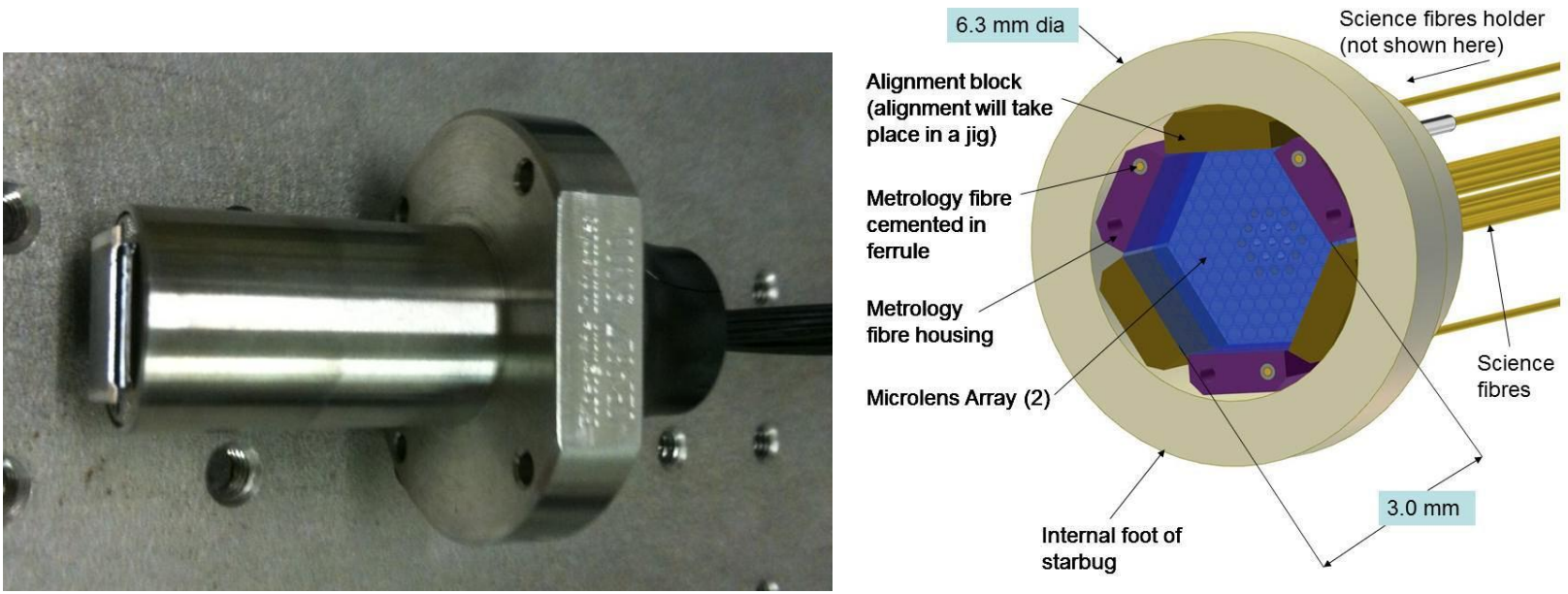

Figure 8. Left: prototype 19-element fiber array with microlens array bonded on to the front (left side of picture). Right: mechanical concept design for microlens array installed in a Starbug unit, from the MANIFEST feasibility study.

\subsection{Sky subtraction}

A key parameter that affects the instrument configuration is the number, location, and configuration of instrument sky fibres. This is particularly important as much of the science with Hector will be for faint galaxy sources or will need to probe the low surface brightness outskirts of brighter galaxies. Correct sky subtraction is therefore essential. The issues are that firstly the sky fibres must be spread out along the slit to sample all the PSF variations within the spectrograph optics to ensure good PCA modes. Secondly, if the sky flux varies over the field either due to telescope/corrector focal ratio variations or from real sky variations then sky must be sampled also across the field. Finally, all of the science fibres will be in IFUs (either hexabundle or microlens based), and thus the sky fibres should also be contained in similar IFUs to avoid the risk of single fibres having different throughput behavior.

There are a number of possible configurations. The currently preferred option is to use a number of sky-fibre IFUs that are not as large as the science IFUs (e.g., 19 rather than 61 fibres). These IFUs will be spread out across the focal plane, across the slit, and across the slits of multiple spectrographs. The advantage of this approach is that these mini-IFUs can also be used on calibration stars in the field (for monitoring seeing and providing input into the post-process atmospheric dispersion correction). The motivation for this approach over a fewer number of science IFUs that can be configured as sky IFUs is that the spectrograph and field variations are better accounted for. The motivation for this approach over a larger number of single fibre IFUs is that this option requires less expansion of the fibre positioning system.

The total number of mini-sky IFUs required will be determined through more careful modeling but will likely be $\sim 35$ for the Hector $3 \mathrm{dF}$ version and $\sim 16$ for the Hector $2 \mathrm{dF}$ version. These numbers represent $\sim 10 \%$ of all fibres dedicated to sky measurement which should ensure good data quality.

\subsection{Spectrographs}

The baseline requirements for the Hector spectrographs are that they should provide a spectral resolution of R 4000 with $\sim 2$ pixel sampling, over a wavelength range from $370-1000 \mathrm{~nm}$, for 3000-7000 fibres of core diameter $\sim 105 \mu \mathrm{m}$. With such a large number of fibres, multiple spectrograph units are required, and thus to keep unit costs manageable there is a strong design preference for a fixed format (i.e., no articulation is allowed for modification of parameter space). Moving components for detector focusing, for example should, however, be allowed.

A key trade study to be conducted for the Hector spectrograph is to examine the cost and performance implications for catadioptric versus transmissive designs. In general, catadioptric (Schmidt-style) spectrographs allow faster cameras, fewer optical components, and superb imaging. The camera speeds can be up to $\sim \mathrm{f} / 1.2$, although there are obstruction losses associated with the fact that the detector must be in the beam. These losses drive the design towards large pupil sizes with an associated increase in cost, although large sized Schmidt correctors are now cheaper and less risky with MRF technology used for fabrication of precision optics. The use of camera elements as the detector Dewar window also adds complexity for catadioptric designs. In general, refractive spectrograph designs allow much smaller beam sizes, which means that individual optical elements are cheaper. However, usually more optical elements are needed to achieve 
the same degree of aberration correction as for a reflective design. Other disadvantages are that for refractive optics high throughput is difficult to achieve in the blue, maximum camera speeds are limited, and scattered light can be problematic.

We have examined strawman designs for reflective spectrographs based on another instrument project, DESpec, with similar requirements to Hector in terms of the fibre core size, the total number of fibres, and the spectral resolution [24]. Figure 9 shows two design options that are based on a fixed format that is similar to the AAOmega configuration. The first is a Schmidt/Schmidt design with a $250 \mathrm{~mm}$ beam, and an F/1.3 camera providing R 4000 over 450-900 nm. Prisms are inserted between the VPHG and the correctors to reduce air-glass surfaces. A "lollipop" style camera Dewar is used to minimize obstruction loss with a field flattener as the Dewar window. The second option uses a double-pass Maksutov design which is much more compact. By placing the detector obstruction close to the pupil, the obstructed region includes the beam area shadowed by the top-end telescope obstruction, and thus losses should be minimized. To increase the wavelength coverage as required by Hector, dual arm versions of both these options will be considered. For refractive spectrograph designs we will consider variants of other replicated spectrographs, such as for MUSE, VIRUS, and the SDSS-based design for WFMOS [25].
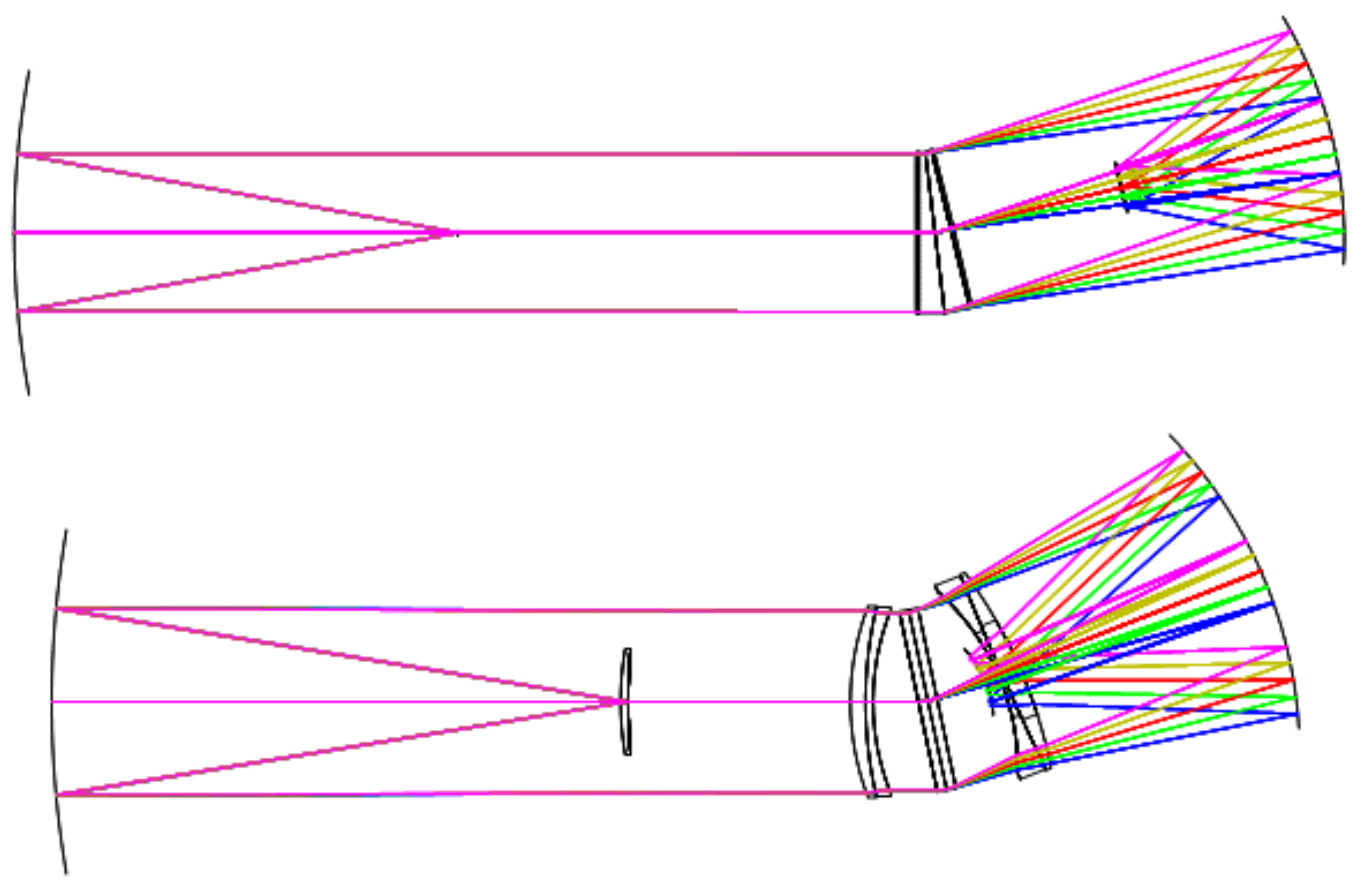

Figure 9. Preliminary optical layouts for a 1-arm catadioptric spectrograph with R 4000 over 450-900 nm for 1000 fibres. The top is a Schmidt/Schmidt design, the bottom is a double-pass Maksutov. For Hector, we would require a 2-armed configuration to extend the wavelength coverage.

\section{CONCLUSIONS}

The Hector instrument is currently at an early design phase. The concept we have outlined in this paper is motivated by the desire to provide an order of magnitude increase over existing facilities in survey capability for spatially resolved galaxy spectroscopy. There are a number of further trade studies that must be conducted to optimize the instrument performance, minimize costs, and to determine the best interface to existing AAT facilities. Studies to be conducted in the next project phase include: the corrector configuration (use of the existing $2 \mathrm{dF}$ or a new $3 \mathrm{dF}$ system), the positioning system technology (whether Starbugs or sequential place robot), the IFU technology (hexabundle versus microlens array), sky subtraction requirements (number and configuration of sky fibres), and the spectrograph configuration (allrefractive versus catadioptric and sub-variants of each). With the solution of these issues, the Hector instrument promises to be a powerful facility that will move into a new region of parameter space for spatially resolved galaxy surveys, providing answers to many currently open questions about galaxy formation and evolution. 


\section{REFERENCES}

[1] Ellis, S.C., et al., "The Millennium Galaxy Catalogue: on the natural subdivision of galaxies", MNRAS 363,1257 (2005).

[2] Bacon, R., et al., "The SAURON project - I. The panoramic integral-field spectrograph", MNRAS 326, 23 (2001).

[3] Cappellari, M., et al., "Constraining the role of star cluster mergers in nuclear cluster formation: simulations confront integral-field data", MNRAS 413, 813 (2011).

[4] Sanchez, S., et al., "CALIFA, the Calar Alto Legacy Integral Field Area survey. I. Survey presentation", A\&A 538, 8 (2012).

[5] Croom, S.M. et al., "The Sydney-AAO Multi-object Integral field spectrograph”, MNRAS 421, 872 (2012).

[6] Sharp, R., et al., "Performance of AAOmega: the AAT multi-purpose fiber-fed spectrograph", Proc. SPIE 6269, 62690G (2006).

[7] Kormendy, J., et al., "Bulgeless Giant Galaxies Challenge Our Picture of Galaxy Formation by Hierarchical Clustering", ApJ 723, 54 (2010).

[8] Kimm, T., et al., "Constraining stellar assembly and AGN feedback at the peak epoch of star formation", MNRAS, in press - astroph/1106.0538, (2012).

[9] Magorrian, J., et al., "The Demography of Massive Dark Objects in Galaxy Centers", AJ 115, 2285 (1998).

[10] Heckman, T., "An optical and radio survey of the nuclei of bright galaxies”, A\&A 87, 152 (1980).

[11] Croton, D., et al., "The many lives of active galactic nuclei: cooling flows, black holes and the luminosities and colours of galaxies", MNRAS 365, 11 (2006).

[12] Sharp, R., \& Bland-Hawthorn, J., "Three-Dimensional Integral Field Observations of 10 Galactic Winds. I. Extended Phase (Myr) of Mass/Energy Injection Before the Wind Blows", ApJ 711, 818 (2010).

[13] Gunn, J., \& Gott, R., “On the Infall of Matter Into Clusters of Galaxies”, ApJ 176, 1 (1972).

[14] Kewley, L., \& Ellison, S., "Metallicity Calibrations and the Mass-Metallicity Relation for Star-forming Galaxies", ApJ 681, 1183 (2008).

[15] Lewis, J., et al., "The Anglo-Australian Observatory 2dF facility”, MNRAS 333, 279 (2002).

[16] Gillingham, P., “A 3 degree prime focus field for the AAT”, Proc SPIE 8446-244 (2012).

[17] Miziarski, S., et al., "Concepts for multi-IFU robotic positioning systems", Proc SPIE 8450-42 (2012).

[18] Gilbert, J., et al., "Starbugs: all-singing, all-dancing fibre positioning robots", Proc SPIE 8450-44 (2012).

[19] Goodwin, M., et al., "MANIFEST instrument concept and related technologies", Proc SPIE 8446-289 (2012).

[20] Bryant, J., et al., "Characterization of hexabundles: initial results”, MNRAS 415, 2173 (2011).

[21] Bryant, J., et al., "Focal ratio degradation in lightly-fused hexabundles", in prep, (2012).

[22] Ellis, S.C., et al., "KOALA: a wide-field, 1000 element integral-field unit for the Anglo-Australian Telescope", Proc SPIE 8446-29 (2012).

[23] Ireland, M., et al., "The AAO's Gemini high-resolution optical spectrograph (GHOS) concept", Proc SPIE 8446-80 (2012).

[24] Saunders, W., "A fast new reflective design for fiber-fed spectrographs", Proc SPIE 8446-196 (2012).

[25] Smee, S., Barkhousera, R., Glazebrook, K., "Design of a multi-object, high throughput, low resolution fiber spectrograph for WFMOS”, Proc SPIE 6269, 62692I (2006). 